\title{
Adaptive Learning Systems and Interference in Causal Inference
}

\author{
ALEXANDER O. SAVI, VU University Amsterdam, the Netherlands \\ NICK TEN BROEKE, University of Amsterdam \& Prowise Learn, the Netherlands \\ ABE D. HOFMAN, University of Amsterdam \& Prowise Learn, the Netherlands
}

\begin{abstract}
Adaptive learning systems can be susceptible to between-subject crosscondition interference by design. This interference has important implications for the implementation and evaluation of A/B tests in such systems as it obstructs causal inference and hurts external validity. We illustrate the problem in an Elo based adaptive learning system, discuss sources and degrees of interference, and provide solutions, using an example in the study of dropout.
\end{abstract}

Additional Key Words and Phrases: adaptive learning systems, a/b testing, cross-condition interference, between-subjects design, dropout

\section{INTRODUCTION}

Learning at scale is slowly but surely gaining ground on its promise to leap education forward. Among the many contributing innovations are two educational technologies: adaptive learning systems and educational $\mathrm{A} / \mathrm{B}$ tests. Using the analogy of a navigation device, the former aims to adapt the learning route to the individual, whereas the latter is used to evaluate new roads [12]. Adaptive learning systems have a long history in artificial intelligence and culminated in various distinct approaches, from intelligent tutoring systems [1] to Elo based systems [9]. Today, adaptive learning systems have nation- and sometimes worldwide reach. A/B tests have their origin in computer science and took major tech companies by storm, with edtech following suit.

In this work we show that the union of both advances can create a challenge: between-subject cross-condition interference. In experimental design, cross-condition interference is primarily recognized as a potential problem for within-subject designs. Within-subject designs create an open path between successive conditions, as subjects visit both. This may for instance create carry-over effects. Many adaptive learning systems are susceptible to such effects in betweensubject designs: user behaviour alters the system and consequently influences other users.

This open path creates an opportunity for cross-condition interference. In causal inference, this is known as a violation of the stable unit treatment value assumption (SUTVA); the assumption that there is no interference between units. Indeed, the response of a particular subject should only depend on the treatment to which it was assigned, and not on the treatment of others. A violation of this assumption obstructs causal inference, a primary reason for the $\mathrm{A} / \mathrm{B}$ testing endeavour. On top of that, interference hurts external validity.

In the following sections, we discuss and illustrate the issue of between-subject cross-condition interference in adaptive learning systems. We do so by (1) formalizing a typical Elo based adaptive learning system on the basis of Prowise Learn, a popular Dutch

Authors' addresses: Alexander O. Savi, o.a.savi@gmail.com, VU University Amsterdam, Amsterdam, the Netherlands; Nick ten Broeke, University of Amsterdam \& Prowise Learn, Amsterdam, the Netherlands; Abe D. Hofman, University of Amsterdam \& Prowise Learn, Amsterdam, the Netherlands. learning platform, (2) describing various sources and degrees of interference in such systems, (3) highlighting a recent dropout study at Prowise Learn to illustrate the problem, and (4) discussing solutions.

\section{ELO BASED ADAPTIVE LEARNING}

\subsection{Prowise Learn}

Prowise Learn started as a research project in 2007, initiated at the University of Amsterdam. In the project, an Elo-based practice platform was developed [5] that aimed to provide researchers with behavioral data of children while they were developing their math proficiency. Following increasing demand, the project was commercialized by spin-off company Oefenweb, which expanded the catalogue with games aimed at practicing language, statistics, and typing skills. In 2018, Oefenweb was branded Prowise Learn after it was acquired by Prowise, a company that develops both hardware and software solutions for education across more than 21 different countries. To date, Prowise Learn hosts and develops 67 games across three different applications, has over 235000 daily users in the Netherlands alone, and garners millions of daily responses ${ }^{1}$.

The large-scale data that are gathered across the different applications of Prowise Learn, provide a unique window into the cognitive development and learning processes of children in both primary and secondary education [e.g., 3]. As such, the system offers researchers with data that can be used to explore a wide variety of research questions. Moreover, the system provides educators with valuable insights into the progress of the children in their classroom, and provides the children with an adaptive learning platform.

\subsection{Adaptive Engine}

To provide all children with relevant content, Prowise Learn tracks both user abilities and item difficulties in real-time. To this end, it combines the Elo algorithm with an explicit scoring rule [6]. Recently, the Elo algorithm has become popular in other adaptive learning systems $[9,14]$, for instance for removing the need to pretest the item bank. The Elo algorithm is an approach in the tradition of artificial intelligence that is self-learning, light-weight, straightforward to use, and that delivers interpretable system parameters.

The scoring rule implemented in Prowise Learn rewards quick correct responses, and is defined as follows:

$$
S\left(x_{i}, t_{i}\right)=x_{i}\left(d_{i}-t_{i}\right)-\left(1-x_{i}\right) P,
$$

where $x_{i} \in 0,1$ is the response accuracy, $t_{i} \in 0, d_{i}$ is the response time and $d_{i}$ is the deadline for the response. This deadline can vary between items (indicated by subscript $i$ ). In the implementation of the model, $d_{i}$ is rescaled such that the deadline equals 1 for each

\footnotetext{
${ }^{1}$ A Prowise Learn demo account can be created here: https://www.prowise.com/en/ prowise-learn/
} 
item. The achieved score equals the remaining time $d_{i}-t_{i}$ for correct responses and a fixed penalty $-P$ for incorrect responses.

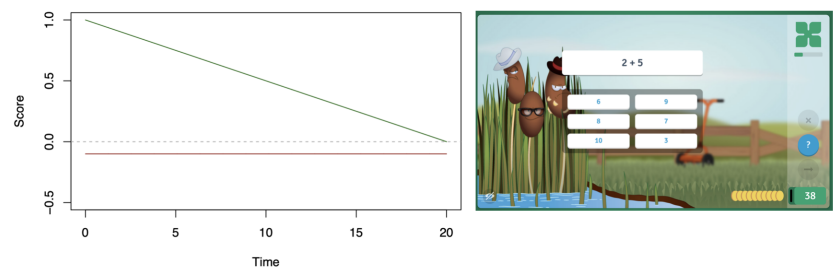

Fig. 1. Left panel: visualization of the scoring rule. Right panel: an addition problem in Prowise Learn.

The scoring rule (Figure 1, left panel) is implemented in a reward system. At each problem attempt a set of coins is visualized, and every second one coin is lost (Figure 1, right panel) until no coins are left. These virtual coins can be collected and spend on virtual trophies.

Using the scoring rule, the difference between the item rating and user rating is related to the expected score of a child given a selected item:

$$
E\left(S \mid \theta_{p}, \beta_{i}\right)=\frac{-P d \xi_{i}^{2} \exp \left(-P \xi_{i}\right)+d \xi_{i} \exp \left(d \xi_{i}\right)-\exp \left(d \xi_{i}\right)+1}{\xi_{i}\left(\exp \left(d \xi_{i}\right)-1+d \xi_{i} \exp \left(-P \xi_{i}\right)\right)}
$$

where $\xi_{i}=\theta_{p}-\beta_{i}$. Following Elo, the item rating and person rating are updated based on the difference between $S$ and $E(S)$ :

$$
\begin{aligned}
& \hat{\theta_{p}}=\hat{\theta_{p}}+K_{p} *(S-E(S)) \\
& \hat{\beta}_{i}=\hat{\beta}_{i}-K_{i} *(S-E(S)),
\end{aligned}
$$

where $K_{p}$ and $K_{i}$ determine the weight of the new information in updating the system parameters. These $K$ values need to be set in such a way that the system adapts sufficiently to incoming data, but also achieves high precision in a period of stability. See [5] for a more detailed description of how the $K$ value and other settings of the adaptive Elo algorithm are specified.

After updating all model parameters, the next item is selected ensuring that the expected probability of a correct response is around $.6, .75$, or .9 , depending of the selected difficulty setting of the child. The child directly receives feedback after every response, and after ten items a game is completed. On the landing page, children can restart the same game or navigate to other games.

\section{A/B INTERFERENCE}

The portrayed Elo based algorithm is illustrative of adaptive systems that provide an open path between different users by design, and as such allow between-subject cross-condition interference between the $\mathrm{A}$ and $\mathrm{B}$ (and $n$ ) of an $\mathrm{A} / \mathrm{B}$ test. The open path is created by the item difficulty updates in Equation 3, as these updates influence the item selection and expected scores for all users.

When subjects change their behaviour following an intervention, this potentially changes their response to an item (expressed in their score $\left.S_{p i}\right)$, subsequently their abilities $\left(\hat{\theta_{p}}\right)$, and ultimately the item difficulties $\hat{\beta}_{i}$ ). When item difficulties change because of an intervention, these changes contaminate the control condition. Vice versa, the control condition might diminish or reverse the potential changes in item difficulties in the treatment condition. This interference can cause item bias (known as differential item functioning in item response theory [8]) between conditions, which obstructs comparability of the ability estimates. It causes a violation of SUTVA, obstructs causal inference, and reduces external validity.

Degrees of Interference. In principle, all A/B tests that directly or indirectly affect the adaptive engine may cause interference. However, the degree of interference depends on the source of interference.

Some $\mathrm{A} / \mathrm{B}$ tests will have a negligible effect on the adaptive engine. For instance, we employed an A/B test to study the effect of a forced problem skipping delay on the amount of exerted effort by the subjects [11]. This subtle intervention only affected a minority of subjects that exploited the problem skipping option, possibly for mining the virtual coins. Moreover, within this group, it only affected a minority of responses.

Other A/B tests may more directly affect the adaptive engine and amplify interference. For instance, we considered evaluating a game in which subjects only practice problems that are at the top of their ability level. This game would have had a more direct influence on the adaptive engine, by means of the item selection function. The game could cause a subject's estimated ability to drop, causing interference through the item difficulty updates.

Then, an extreme case is an A/B test that directly alters the adaptive engine. For instance, we considered evaluating the scoring rule itself. The scoring rule directly impacts the item difficulties, creating the additional difficulty that user abilities and item difficulties become incompatible.

In the following sections, we portray a recent study on dropout in Prowise Learn, discuss how a follow-up A/B test would suffer from interference, and finally discuss solutions.

\section{ILLUSTRATION: DROPOUT}

\subsection{Background}

Dropout, sometimes referred to as attrition or simply quitting, is a central problem in adaptive (and other) online learning systems. These systems typically give students more flexibility than traditional, brick-and-mortar education, creating an additional challenge for students to persist on their academic tasks. Dropout within these systems is severe-by some estimates as high as $90 \%$ [13]-and is not going unnoticed [e.g., 2].

An internal analysis of dropout in Prowise Learn applications uncovered an estimate of $30 \%$ of the games that are not completed. Such behavior is problematic as it impedes the system to function optimally, thereby negatively affecting the learning progress of the users. Figure 2 depicts the negative association between the average growth of 16534 Prowise Learn users over a period of two months, and the proportion of games that they did not finish $(r=-0.21,95 \% C I[-0.22,-0.2], p<.001)$. This underlines the importance of reducing dropout behavior in adaptive systems like Prowise Learn. 


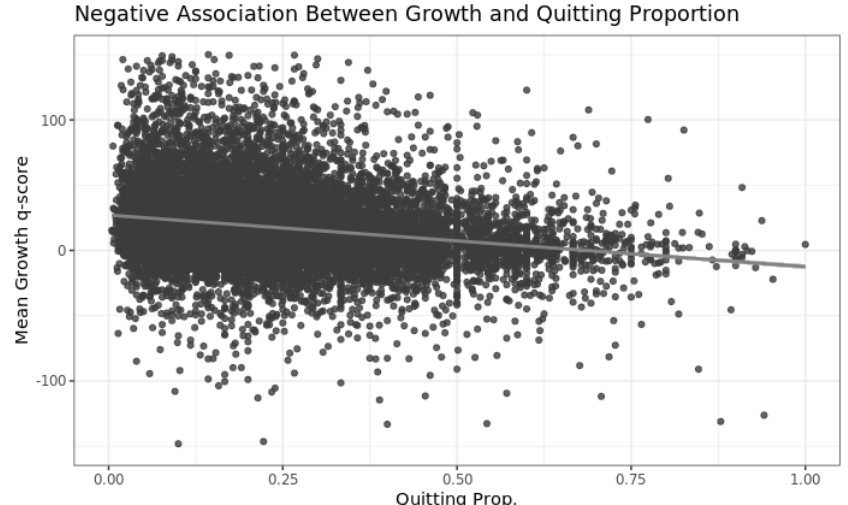

Fig. 2. Mean growth in two months ( $y$-axis) for proportions of uncompleted sessions ( $x$-axis). Each dot represents a unique user. Only users who played at least ten games in the last two months are included.

In order to understand dropout behavior, we fitted a multi-state survival model. Multi-state models extend traditional survival models by allowing more transient states than the two traditional 'alive' and 'dead' states. We defined three states. For any given game, each user started in a persisting state (state 1). A user that did not finish a game and started a new game within the same session, was moved to the soft-quit state (state 2). The user remained in this state until either finishing a game in its entirety (resulting in a move back to state 1) or until the game was quit before completion and the session was ended (resulting in a move to the hard-quit state 3) The hard-quit state was an absorbing state as the system did not allow to exit this state once entered. If a user was already in state 1 and ended the playing session without completing the last game, it also resulted in a move to state 3 . If a user ended the session after completing an entire game, it ended in state 1 and thus no hard-quit occurred in that session. This framework corresponds to the following transition intensity matrix, which denotes the instantaneous risk associated with each state transition:

$$
\left[\begin{array}{ccc}
-\left(q_{12}+q_{13}\right) & q_{12} & q_{13} \\
q_{21} & -\left(q_{21}+q_{23}\right) & q_{23} \\
0 & 0 & 0
\end{array}\right]
$$

where the diagonal entries ensure that the rows in the matrix sum to zero, which is a necessary condition for a matrix to be defined as a transition intensity matrix [7]. The null entries indicate that a transition is not possible.

This allowed us to investigate the influence of a set of covariates on each transition intensity, by maximizing the likelihood with regards to the transition intensities and the $\beta$ parameters in

$$
q_{h j}(z(t))=q_{h j}^{0} e^{\beta^{T} z(t)},
$$

which should be read as: the state transition $q_{h j}$, with a covariate vector of the form $z(t)$, is the product of the baseline intensity function $q_{h j}^{0}$ and the influence of each covariate value, quantified by the $\beta$ vector. This $\beta$ vector is exponentiated to yield hazard ratios that indicate the relative importance of each covariate in decreasing or increasing the risk of state transition $q_{h j}$.

Different covariates were investigated, for instance the difficulty setting used by a user, whether someone was playing outside of school hours, or whether a user exhibited post-error slowing. Interestingly, we found that making three errors in a row is most predictive of a quit, compared to making any other series of mistakes, and compared to any other covariate. Figure 3 shows how the likelihood of a quit increases with each sequential error (quantified by means of odds ratios) and how this risk is attenuated when a user exceeds this threshold.

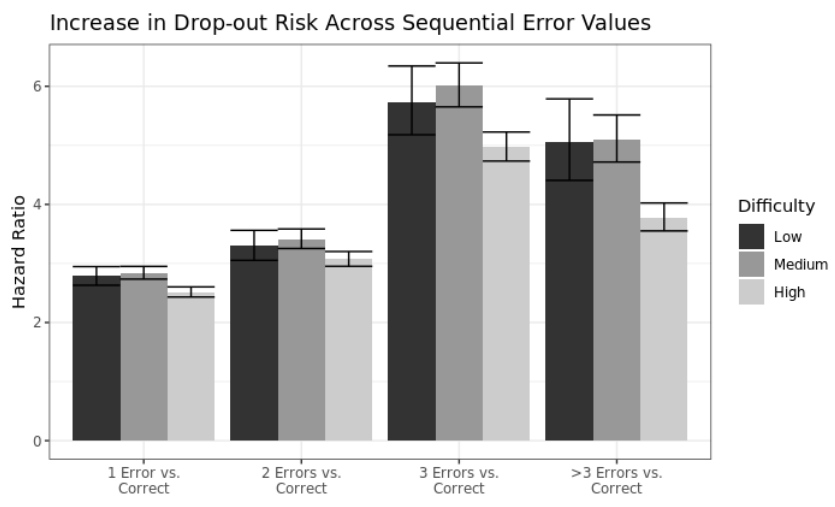

Fig. 3. Odds ratios associated with each sequential error value, split by difficulty setting. Odds ratios are in comparison with a correct response.

\subsection{A/B Interference in a Sequential Error Intervention}

Following this three-strikes-out result, we consider an A/B test to determine the effect of selecting an easy item-given $\hat{\theta_{p}}$-whenever a user makes two mistakes in a row. This intervention increases the probability of a correct response, breaking the chain of sequential errors.

Now, imagine that a user in the treatment condition submits two sequential incorrect responses and a relatively easy third item is selected. Such an item might elicit a different response process than normal, for instance caused by distraction or a lack of motivation. This could cause the observed score $S$ to deviate from the expected score $E(S)$, which will push the item rating upwards or downwards. If this happens many times, it introduces unwanted bias in the estimates of $\hat{\beta}_{i}$. Then, if this very item is selected for a user in the control condition, this user is likely to have a true expected score for this item that is lower or higher than the system assumes, which will in turn introduce bias in the estimate of $\hat{\theta_{h}}$. Thus, provided that the sequential error intervention indeed elicits a different response process for the targeted items, this effect is spills into the control condition, violating SUTVA and jeopardizing our ability to conduct valid inference over our $\mathrm{A} / \mathrm{B}$ test.

\section{SOLUTIONS}

We distinguish three implementation scenarios. 


\subsection{Joint}

In the joint scenario, all conditions of an $\mathrm{A} / \mathrm{B}$ test jointly run on top of the same instance of the adaptive engine, and interference is allowed. As discussed, the expected cross-condition interference obstructs causal inference and introduces unwanted bias in the system's parameters.

If this joint scenario is the only feasible option, monitoring interference using offline data can provide insights into the degree of interference. This involves reanalysing the collected data for each $\mathrm{A} / \mathrm{B}$ test condition. Item parameters can be estimated offline, using a subset of the collected data. Deviations between the online item parameters and the re-estimated item parameters in both conditions should be inspected. This can be done on a daily basis (depending on how much data is collected during the A/B test), and a significant unwanted impact on the item ratings can be used as a stopping criterion for the $\mathrm{A} / \mathrm{B}$ test. If there is evidence for significant interference, one should consider the completely disjoint scenario, or the more pragmatic partially disjoint scenario.

\subsection{Partially disjoint}

In the partially disjoint scenario, all conditions of an A/B test jointly run on top of the same instance of the adaptive engine, but the path between conditions is barred. In the Elo-algorithm, this involves temporarily disabling the item difficulty update function (Equation 3) in the treatment condition. In this scenario, the item parameters are still updated as usual by the responses of both the subjects in the control condition and the users not included in the A/B test. However, it removes the possibility for subjects in the treatment condition to introduce unwanted bias and contaminate the control condition.

Although interference is barred, this scenario elicits a problem if the treatment condition significantly impacts the adaptive engine. The item ratings will reflect the altered response processes in the treatment condition less and less, as the item ratings are updated according to the responses in the control condition. Ability ratings that are estimated on the basis of the such incompatible item ratings cannot be trusted. This holds just as much for possible offline reanalyses.

Ultimately, if there is no meaningful difference between the item parameter trajectories in both conditions and a significant difference between conditions is found, it should generally be safe to conclude that the treatment had an effect while the adaptive engine was not impacted.

\subsection{Completely disjoint}

In the completely disjoint scenario, the conditions of an $\mathrm{A} / \mathrm{B}$ test run on different instances of the adaptive engine. This scenario involves running parallel systems, only varying with respect to the treatment It is the most versatile scenario and interference is fully eradicated.

To conduct the sequential error intervention $\mathrm{A} / \mathrm{B}$ test in the completely disjoint scenario would be ideal, as it allows one to negate the possibility of interference, retain external validity, and ensure that no incompatibility issues between item difficulties and response processes can arise. However, there is a practical downside. For commercial, large scale systems like Prowise Learn, this solution is often not feasible. It takes too much effort and resources to clone the entire system, run the $\mathrm{A} / \mathrm{B}$ test, and then attempt to merge the two systems back into one, without impacting the experience of both users and teachers.

\section{DISCUSSION}

In this work, we highlighted interference scenarios in adaptive learning systems that are created by design. Additionally, in vivo experiments such as A/B tests can create many other open paths between conditions that obstruct causal inference and reduce external validity. Think of two friends in a classroom, jointly working on their own adaptive problem sets, while being assigned to different conditions of an $\mathrm{A} / \mathrm{B}$ test.

On the other hand, not all $\mathrm{A} / \mathrm{B}$ tests in adaptive learning systems suffer from the discussed interference. Not all $\mathrm{A} / \mathrm{B}$ tests are expected to affect the adaptive engine in any sense, including most of the studies that target 'clicking' rather than learning [10].

Finally, we must note that we assume that item difficulties are converged before the start of an A/B test. This is not an easy to test requirement [4], but crucial to assess in the disjoint scenarios, to guarantee that changes in the adaptive engine are solely caused by the $\mathrm{A} / \mathrm{B}$ test.

\section{REFERENCES}

[1] J. R. Anderson, C. F. Boyle, and B. J. Reiser. 1985. Intelligent Tutoring Systems Science 228, 4698 (apr 1985), 456-462. https://doi.org/10.1126/science.228.4698.456

[2] Fisnik Dalipi, Ali Shariq Imran, and Zenun Kastrati. 2018. MOOC dropout prediction using machine learning techniques: Review and research challenges. In 2018 IEEE Global Engineering Education Conference (EDUCON). IEEE, 1007-1014.

[3] Abe Dirk Hofman et al. 2018. Psychometric analyses of computer adaptive practice data: A new window on cognitive development. Universiteit van Amsterdam [Host].

[4] Abe D Hofman, Matthieu JS Brinkhuis, Maria Bolsinova, Jonathan Klaiber, Gunter Maris, and Han LJ van der Maas. 2020. Tracking with (Un)Certainty. Fournal of Intelligence 8, 1 (2020), 10

[5] S. Klinkenberg, M. Straatemeier, and H.L.J. van der Maas. 2011. Computer adaptive practice of Maths ability using a new item response model for on the fly ability and difficulty estimation. Computers \& Education 57, 2 (sep 2011), 1813-1824. https://doi.org/10.1016/j.compedu.2011.02.003

[6] Gunter Maris and Han van der Maas. 2012. Speed-Accuracy Response Models: Scoring Rules based on Response Time and Accuracy. Psychometrika 77, 4 (sep 2012), 615-633. https://doi.org/10.1007/s11336-012-9288-y

[7] Luís Meira-Machado, Jacobo de Uña-Álvarez, Carmen Cadarso-Suárez, and Per K Andersen. 2009. Multi-state models for the analysis of time-to-event data. Statistical methods in medical research 18, 2 (2009), 195-222.

[8] Gideon J Mellenbergh. 1989. Item bias and item response theory. International journal of educational research 13, 2 (1989), 127-143.

[9] Radek Pelánek. 2016. Applications of the Elo rating system in adaptive educational systems. Computers \& Education 98 (jul 2016), 169-179. https://doi.org/10.1016/j. compedu.2016.03.017

[10] Justin Reich. 2015. Rebooting MOOC Research. Science 347, 6217 (jan 2015), 34-35 https://doi.org/10.1126/science.1261627

[11] Alexander O. Savi, Nienke M. Ruijs, Gunter K.J. Maris, and Han L.J. van der Maas. 2018. Delaying access to a problem-skipping option increases effortful practice: Application of an A/B test in large-scale online learning. Computers \& Education 119 (apr 2018), 84-94. https://doi.org/10.1016/j.compedu.2017.12.008

[12] Alexander O. Savi, Han L. J. van der Maas, and Gunter K. J. Maris. 2015. Navigating Massive Open Online Courses. Science 347, 6225 (feb 2015), 958-958. https: //doi.org/10.1126/science.347.6225.958

[13] Colin Taylor, Kalyan Veeramachaneni, and Una-May O'Reilly. 2014. Likely to stop? predicting stopout in massive open online courses. arXiv preprint arXiv:1408.3382 (2014).

[14] Michael Yudelson, Yigal Rosen, Steve Polyak, and Jimmy de la Torre. 2019 Leveraging Skill Hierarchy for Multi-Level Modeling with Elo Rating System. In Proceedings of the Sixth (2019) ACM Conference on Learning @ Scale. ACM. https://doi.org/10.1145/3330430.3333645 